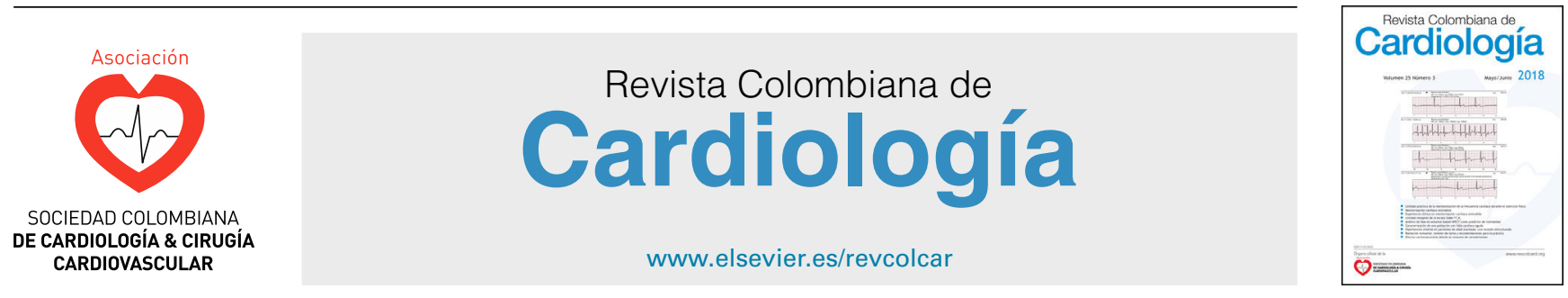

CARDIOLOGÍA DEL ADULTO - PRESENTACIÓN DE CASOS

\title{
Elevación persistente de troponina I
}

\section{María Lasala Alastueya,*, Beatriz García Rodríguez ${ }^{\mathrm{b}}$, María Rosa Gracia Matilla ${ }^{\mathrm{b}}$, Carlos Rubén López Perales ${ }^{a}$ y Elena Rivero Fernández ${ }^{a, b, c}$}

\author{
a Servicio de Cardiología, Hospital Miguel Servet, Zaragoza, España \\ b Servicio de Bioquímica, Hospital Miguel Servet, Zaragoza, España \\ c Servicio de Cardiología, Hospital San Jorge de Huesca, Huesca, España
}

Recibido el 19 de noviembre de 2018; aceptado el 19 de marzo de 2019

Disponible en Internet el 11 de julio de 2019

\section{PALABRAS CLAVE \\ Troponina I; Interferencia analítica; Reacción falsa positiva}

\section{KEYWORDS}

Troponin I; Analytical interference; False positive reaction

\begin{abstract}
Resumen La troponina cardiaca es el marcador bioquímico más sensible y específico de daño/necrosis miocárdica, de ahí que desempeñe un papel crucial en el diagnóstico del síndrome coronario agudo. Sin embargo, en ocasiones, como en el caso clínico que se describirá, la elevación anormal de troponina no siempre obedece a un síndrome coronario agudo trombótico, sino a causa cardiaca sin enfermedad coronaria significativa, causa extracardiaca o alteración analítica (verdaderos falsos positivos). El interés de este caso radica en que siempre debería tenerse en mente la posibilidad de que se produzca un falso positivo de troponina por causa analítica, en especial en situaciones clínicas sin una razón obvia de daño miocárdico y cuando no sea evidente la confirmación de daño miocárdico mediante pruebas complementarias.

๑) 2019 Sociedad Colombiana de Cardiología y Cirugía Cardiovascular. Publicado por Elsevier España, S.L.U. Este es un artículo Open Access bajo la licencia CC BY-NC-ND (http:// creativecommons.org/licenses/by-nc-nd/4.0/).
\end{abstract}

\section{Persistent elevation of troponin I}

Abstract Cardiac troponin is the most sensitive and specific biochemical marker for myocardial damage / necrosis, and thus has a crucial role in the diagnosis of acute coronary syndrome. However, occasionally, as in the clinical case that will be described, the abnormal elevation of troponin does not always obey that of an acute coronary syndrome, but also to a cardiac cause with no significant coronary disease, extra-cardiac cause, or analytical change (true false positives). The interest in this case lies in that it should always be borne in mind that a false positive

\footnotetext{
* Autor para correspondencia.

Correo electrónico: mlasalaalastuey@gmail.com (M. Lasala Alastuey).
} 
troponin can be produced due to an analytical cause. This can be the case in clinical situations with no obvious reason for myocardial damage and when the confirmation of myocardial damage may not be evident using complementary tests.

(c) 2019 Sociedad Colombiana de Cardiología y Cirugía Cardiovascular. Published by Elsevier España, S.L.U. This is an open access article under the CC BY-NC-ND license (http:// creativecommons.org/licenses/by-nc-nd/4.0/).

\section{Introducción}

La troponina cardiaca es el marcador bioquímico más sensible y específico de daño/necrosis miocárdica. El Joint ESC Task Force for the Universal Definition of Myocardial Infarction defiende que el diagnóstico de infarto agudo de miocardio se basa en un aumento o descenso de la troponina dentro de un contexto clínico apropiado ${ }^{1}$. En consecuencia, el valor de la troponina desempeña un rol fundamental en el diagnóstico y manejo del síndrome coronario agudo y tiene gran significado médico y científico.

\section{Caso}

Varón de 64 años, con factores de riesgo cardiovascular (hipertensión arterial, diabetes mellitus tipo 2, dislipidemia y exfumador), quien ingresó al servicio de Cardiología por episodio de opresión centrotorácica en hemitórax izquierdo, sin cambios electrocardiográficos sugestivos de isquemia, pero con elevación de troponina I (Tn I) de 1,33 ng/ml (DXI Beckman Cokulter, límite alto de la normalidad 0,04 $\mathrm{ng} / \mathrm{ml}$ ), sin objetivarse curva enzimática, pero realizando solo tres determinaciones (1,2-1,3-1,3 $\mathrm{ng} / \mathrm{ml})$. Se hizo ecocardiograma en el cual no se encontraron alteraciones de la contractilidad, función normal del ventrículo izquierdo, insuficiencia mitral ligera por prolapso de $\mathrm{P} 2$, sin otros hallazgos. En coronariografía se evidenciaron arterias coronarias con flujo lento, sin lesiones angiográficas significativas, con irregularidades en DA media y CD proximal. Se pautó tratamiento médico de síndrome coronario agudo sin elevación del ST con doble antiagregación durante un año. Desde el alta permaneció asintomático, en clase funcional I de acuerdo con la NYHA. Un mes más tarde ingresó por episodio de dolor punzante a nivel centrotorácico, de intensidad moderada y cese en dos minutos, habiendo tomado un nitrato sublingual. En urgencias, sin dolor torácico, se objetivó en ECG ausencia de alteraciones de la repolarización. En análisis de sangre se detectó troponina I elevada de 1,34$1,36 \mathrm{ng} / \mathrm{ml}$ en dos determinaciones extraídas con 6 horas de diferencia. Ingresó para estudio. Se trataba de un dolor torácico con rasgos atípicos, elevación de Tnl en meseta sin curva enzimática, de modo que se revisó coronariografía sin lesiones significativas y se repitió ecocardiograma, que no mostró cambios respecto al previo. Se planteó la posibilidad de que la elevación de troponina I cardiaca pudiera estar justificada en este caso por otros motivos, puesto que se obtenían siempre cifras similares de troponina I en análisis hechos con diferencia de más de un mes de tiempo.
Tabla 1 Causas de elevación de troponina

Síndrome coronario agudo

Insuficiencia cardiaca

Accidente cerebrovascular

Tromboembolia pulmonar

Disección aguda de aorta

Taquiarritmias

Shock/hipotensión

Sepsis

Miopericarditis

Endocarditis

Miocardiopatía de takotsubo

Contusión cardiaca

Cardiotoxicidad por quimioterapia

Ablación por radiofrecuencia

Drogas simpaticomiméticas

Basada en artículo de Thygesen K, Mair J, Katus H, Plebani M, Venge $\mathrm{P}$, Collinson $\mathrm{P}$, et al. Recommendations for the use of cardiac troponin measurement in acute cardiac care. Eur Heart J. 2010;31:2197-206.

La troponina cardiaca puede elevarse por las siguientes razones (tabla 1$)^{1,2}$ :

- Enfermedad coronaria significativa de causa isquémica.

- Causa cardiaca pero sin enfermedad coronaria significativa.

- Síndrome coronario agudo de causa no aterosclerótica (por ejemplo espasmo coronario, embolia coronaria, anemia, arritmias, hipertensión, hipotensión, entre otras).

- Otras causas.

- Causa extracardíaca.

- Alteración analítica: verdaderos falsos positivos.

Por tanto, la elevación anormal de troponina (T o I) se relaciona con lesión miocárdica, pero no siempre indica la causa precisa, y es común en muchas enfermedades, sin indicar necesariamente la presencia de un síndrome coronario agudo trombótico ${ }^{3,4}$. En este caso se valoró la posibilidad de que se tratara de algún tipo de enfermedad extracardíaca (tabla 1) que pudiera justificar una elevación persistente de troponina I, sin curva enzimática y sin síntomas de origen cardiaco. Se hizo resonancia cardiaca, compatible con la normalidad, en la que no se evidenciaron signos de miocarditis o de otras miocardiopatías ni signos de isquemia aguda o subaguda. Se practicaron serologías y estudio de autoinmunidad, sin hallazgos. En vista de los datos obtenidos, se 
sospechó que se pudiera tratar de una interferencia analítica, por lo que se contactó con el servicio de Bioquímica Clínica.

Puede haber diferentes tipos de interferencia analítica en los inmunoensayos para la determinación de troponina; los más frecuentes son los siguientes ${ }^{3-5}$ :

- Interferencia por coágulos de fibrina: se trata de un hecho aislado y puntual, y existe baja probabilidad de que se repita en el mismo paciente. Se cree que la interferencia puede deberse a la unión inespecífica con la fibrina del anticuerpo o bien porque utiliza más volumen de muestra del recomendado para la técnica y, por tanto, el resultado de la troponina puede resultar aumentado de manera falsa. Se puede corregir este error esperando a que se produzca una buena retracción del coágulo antes de la centrifugación e incrementando el tiempo de centrifugación de las muestras.

- Interferencia por anticuerpos heterófilos: el suero de algunos pacientes contiene anticuerpos heterófilos endógenos humanos capaces de unirse a otras inmunoglobulinas. En general, el término anticuerpos heterófilos engloba al grupo de anticuerpos heterófilos, anticuerpos humanos antianimal (HAMA), anticuerpos terapéuticos, factor reumatoide y otros autoanticuerpos. Estos anticuerpos se pueden adquirir por causa iatrogénica o no iatrogénica (uso de anticuerpos monoclonales de ratón para tratamientos oncológicos o en pruebas de imagen, transfusiones sanguíneas, vacunación contra enfermedades infecciosas, cuidado de animales, entre otras). La prevalencia de los anticuerpos heterófilos en la población varía entre 0,2 y $40 \%{ }^{4}$.

El efecto de los anticuerpos heterófilos presentes en el suero de algunos pacientes en los diferentes inmunoanálisis se ha descrito con anterioridad y los inmunoanálisis que miden la troponina no son la excepción, ya que pueden ser afectados por este tipo de interferencia ${ }^{6}$. Para evitar esta interferencia el laboratorio de Bioquímica puede hacer la determinación de troponina mediante un ensayo de otra casa comercial, que emplee distintos anticuerpos, o bien usar bloqueantes para estos anticuerpos heterófilos.

- Interferencia por fosfatasa alcalina: una muestra con una elevada concentración o actividad de fosfatasa alcalina puede producir un resultado incorrecto en aquellos ensayos que emplean fosfatasa alcalina como amplificador de la señal. Este exceso de actividad o concentración de fosfatasa alcalina del paciente produce un aumento de señal, que se traduce en mayor concentración de troponina6.

Para la determinación de troponina I se utilizó un ensayo inmunoenzimático (Access AccuTnl +3, Beckman Coulter) de dos posiciones ("tipo sandwich"). En este ensayo se emplean partículas paramagnéticas recubiertas con anticuerpo monoclonal anti-Tnl ("anticuerpo de captura"), a las que se unirán las moléculas de troponina y un anticuerpo monoclonal anti-Tnl ("anticuerpo marcador"') conjugado con fosfatasa alcalina, que se añade tras una fase de lavado y reacciona con distintos sitios antigénicos de unión de las moléculas troponina I capturadas, lo cual da una señal visible que se puede medir y cuantificar. Cuando existen anticuerpos heterófilos, específicos para los anticuerpos anti-Tnl que utiliza el ensayo, se unen en ausencia de analito, dando una señal detectable y provocando un falso positivo para troponina I.

En este caso, tras descartar factor reumatoide, se comunicó al fabricante del ensayo una concentración elevada de fosfatasa alcalina y coágulos de fibrina en la muestra del paciente, y se envió una muestra de sangre del mismo al laboratorio externo (Beckman Coulter, Marsella) para determinar la posible presencia de anticuerpos. Para ello, se utilizaron distintos tipos de bloqueantes de anticuerpos heterófilos (PolyMak 33, HBR-1) y relacionados de fosfatasa alcalina (AP Mutein). Se realizó la medición de troponina antes y después del tratamiento de la muestra con bloqueantes y se comprobó la existencia de una interferencia analítica debida a la actividad de la fosfatasa alcalina, obteniendo un valor real de troponina I de 0,01 cuando se añadió el bloqueante (AP Mutein).

En conclusión, la posibilidad de que se produzca un falso positivo de troponina por causa analítica debería tenerse siempre en mente, especialmente en situaciones clínicas sin una causa obvia de daño miocárdico y cuando la confirmación de daño miocárdico mediante pruebas complementarias (ECG, ecocardiograma, resonancia cardiaca, coronariografía u otras pruebas complementarias) no es evidente $\mathrm{e}^{3,4}$, puesto que puede conllevar consecuencias clínicas importantes en cuanto a tratamientos y pruebas diagnósticas efectuadas al paciente.

\section{Financiación}

Ninguna.

\section{Conflicto de intereses}

Ninguno.

\section{Bibliografía}

1. Thygesen K, Mair J, Katus H, Plebani M, Venge P, Collinson P, et al. Recommendations for the use of cardiac troponin measurement in acute cardiac care. Eur Heart J. 2010;31:2197-206.

2. Koracevic G, Cosic V, Stojanovic I. False positive troponin - a true problem. J Med Biochem. 2013;32:197-206.

3. Lum G, Solarz DE, Farney L. False positive cardiac troponin results in patients without acute myocardial infarction. Lab Med. 2006;37:546-50

4. Legendre-Bazydlo L, Haverstick DM, Kennedy JLW, Dent JM, Bruns DE. Incremento persistente de troponina I cardiaca en plasma sin evidencia de lesión cardiaca. Clin Chem. 2010;56:702-7.

5. Chueca Rodríguez MP, Güell Miró R, Gelabert Orench JM, Izquierdo Quince F. Comisión de interferencias y efectos de los medicamentos. sociedad española de bioquímica clínica y patología molecular. Interferencias en la medición de troponina. Química Clínica. 2007;26:216-23.

6. Herman DS, Ranjitkar P, Yamaguchi D, Grenache DG, Greene DN. Endogenous alkaline phosphatase interference in cardiac troponin I and other sensitive chemiluminescence immunoassays that use alkaline phosphatase activity for signal amplification. Clin Biochem. 2016;49:1118-21. 